\title{
Fomepizole in the treatment of acute methanol poisonings: Experience from the Czech mass methanol outbreak 2012-2013
}

\author{
Sergey Zakharov ${ }^{a}$, Tomas Navratili ${ }^{b}$ Daniela Pelclova ${ }^{a}$
}

\begin{abstract}
Objective. During an outbreak of mass methanol poisonings in the Czech Republic in 2012-2013, fomepizole was applied as an alternative antidote to ethanol. We present the laboratory data, clinical features, adverse reactions, and treatment outcomes in all patients treated with fomepizole.

Methods. Combined retrospective and prospective case series study in 25 patients, median age 50 (16-73) years, 18 males and 7 females.

Results. There were $24 \%$ fatalities, $36 \%$ survivors without health impairment, and $40 \%$ survivors with sequelae. All the patients who died were comatose on admission; the mortality was $50 \%$ among patients in a coma. The median intensive care unit length of stay was six (2-22) days. The median total dose of fomepizole was 2 (1-9) g. Complications were observed in 7/25 cases: aspiration pneumonia (4), sepsis (2), bleeding (2), malignant arrhythmia (1), delirium tremens (1), and rebound of acidosis (1). The patients who survived without impairment were less acidotic than those who died or survived with sequelae $(P<0.01)$. No difference in serum methanol and formate was found between the three groups. Conclusion. There is no evidence whether fomepizole is a more efficient antidote than ethanol with regards to the hospital mortality. The possibility of delirium tremens in the patients with a history of chronic alcohol abuse has to be taken in consideration. The benefits of fomepizole were indirect: no need to monitor serum ethanol's level during the hemodialysis in severely poisoned patients and less working overload on ICU doctors treating several poisoned patients simultaneously.
\end{abstract}

Key words: methanol poisoning, fomepizole, ethanol, treatment outcome, visual sequelae, CNS sequelae

Received: July 31, 2014; Accepted with revision: October 15, 2014; Available online: November 6, 2014 http://dx.doi.org/10.5507/bp.2014.056

${ }^{a}$ Toxicological Information Center, Department of Occupational Medicine, $1^{\text {st }}$ Faculty of Medicine, Charles University in Prague and General University Hospital, Prague, Czech Republic

${ }^{b}$ Department of Biomimetic Electrochemistry, J. Heyrovsky Institute of Physical Chemistry of AS CR, Prague Corresponding author: Sergey Zakharov, e-mail: Sergey.Zakharov@vfn.cz

\section{INTRODUCTION}

Acute methanol poisoning is a serious cause of outbreaks of mass poisonings resulting in serious health sequelae and high mortality ${ }^{1,2}$. These poisonings generally occur either intentionally through abuse, attempted suicide or unintentionally through misuse or accident ${ }^{3}$. It is a medical emergency where rapid administration of antidotes, fomepizole or ethanol, preventing toxic metabolite formic acid formation by blocking alcohol dehydrogenase $(\mathrm{ADH})$ is crucial for successful treatment ${ }^{4,5}$. Formate anions as the products of methanol metabolism have a strong cytotoxic effect by inhibition of mitochondrial respiration $^{6,7}$. The accumulation of formic acid results in metabolic acidosis, lactacidemia, visual impairment and damage to the basal ganglia, especially when the concentration rises to $9-11 \mathrm{mmol} / \mathrm{L}$ (approximately 300$400 \mathrm{mg} / \mathrm{L})\left(\right.$ ref. $\left.^{8-11}\right)$.

The role of ethanol in the treatment of acute methanol poisonings is well-established ${ }^{12,13}$. Ethanol has approximately ten times higher affinity for ADH than methanol. Thus, it effectively blocks the enzyme when its concentration in blood serum is between $1000-1500 \mathrm{mg} / \mathrm{L}$ (22$33 \mathrm{mmol} / \mathrm{L})\left(\right.$ ref. $\left.^{5,14}\right)$. Fomepizole (4-methylpyrazole) is another effective antidote with affinity for ADH several thousand times higher than of methanol ${ }^{15-18}$.

There is some evidence for the superiority of fomepizole in the treatment of methanol-poisoning ${ }^{16-22}$. Several authors have reported that fomepizole is pharmacokinetically more predictable than ethanol, has a safer sideeffect profile, and reduces the need for hemodialysis ${ }^{19-23}$. However, few clinical studies exist to support or refute these arguments, and the evidence favoring fomepizole over ethanol consists of a few small, non-comparative trials involving a total of 51 patients and none of them was prospective ${ }^{2,18,22}$. Based on the most comprehensive current systematic assessment of the relevant literature, Beatty L. et al. urged further research into the relative benefits of fomepizole in the management of toxic alcohol ingestion ${ }^{19}$.

In this study, we report data based on the recent mass methanol poisoning in the Czech Republic in 2012-2013 (ref. ${ }^{24}$ ). The antidote fomepizole was recently (2013) added to the WHO Essential Medicines List, but its availability is still limited. Until September 2012, fomepizole was not registered in the Czech Republic. On September $12^{\text {th }}$, one week after the first cases of methanol poisonings emerged, the Czech Toxicological Information Center 
(TIC) asked the Ministry of Health for emergency permission to distribute fomepizole to the hospitals treating the poisoned patients. The Ministry issued permission on the same day, and from the following day, fomepizole was supplied to 16 hospitals in 10 regions of the Czech Republic.

We performed a combined retrospective and prospective case series study in 25 methanol-poisoned patients treated with fomepizole in order to present the new data for the laboratory parameters, clinical features, adverse reactions, treatment measures and outcomes, which will be useful for the further meta-analysis studies of the efficacy, safety and cost-effectiveness of treatment of acute methanol poisonings with fomepizole.

\section{MATERIALS AND METHODS}

\section{Patients and procedures}

The study was designed as a combined retrospective and prospective case series study. A total of 25 cases of confirmed methanol poisonings were treated with fomepizole during the period from 14 September 2012 until 12 July 2013. All these poisonings have been documented using a standardized admission protocol developed after the Norwegian methanol outbreak ${ }^{2}$, and the discharge reports of these patients with the results of the neurological and ophthalmologic examinations on admission, during hospitalization, and on discharge have been collected and analyzed in the Czech TIC. A detailed medical record of the history of the poisoning, the onset, and the dynamics of the signs and symptoms of ocular and systemic toxicity were obtained either directly from the patients or from the relatives of critically ill persons on admission, and specified retrospectively in standardized questionnaires.

On admission, the laboratory investigations included serum concentrations of methanol, ethanol, formate, lactate, electrolytes, arterial blood gases, anion and osmolal gaps, glucose, renal- and hepatic tests, complete blood count, hematocrit, and serum proteins. The diagnosis was established if (1) a history of recent ingestion of illicit spirit was available, and serum methanol concentration was more than $6.2 \mathrm{mmol} / \mathrm{L}(20 \mathrm{mg} / \mathrm{dL})$, and/or an osmolal gap higher than $19 \mathrm{mOsm} /\left(\mathrm{kg} \mathrm{H}_{2} \mathrm{O}\right)$ were noted, or (2) there was a history/clinical suspicion of methanol poisoning, serum methanol detectable, and at least two of the following were present: $\mathrm{pH}$ less than 7.3 , serum bicarbonate less $20 \mathrm{mmol} / \mathrm{L}(20 \mathrm{mEq} / \mathrm{L})$, and anion gap more than $19 \mathrm{mmol} / \mathrm{L}(19 \mathrm{mEq} / \mathrm{L})$.

In 11 patients (58\% of survivors), the follow-up examination was fulfilled three to six months after discharge from hospital within the prospective study of long-term visual and CNS sequelae of acute methanol poisonings ${ }^{25}$. The clinical examination protocol included complete ocular examination and standard ophthalmic tests (visual acuity, perimeter, color vision assessment, contrast sensibility, fundus examination), optical coherence tomography (OCT) with retinal nerve fibers layer thickness evaluation, visual evoked potentials (VEP), magnetic resonance imaging of the head, neurological and neuropsychological examinations, biochemical tests (electrolytes, glucose, glycohemoglobin, albumin, pre-albumin, renal and hepatic tests, cholesterol, lipids, thyroid-stimulating hormone (TSH), vitamin $\mathrm{B}_{12}$, carbohydrate deficient transferrin (CDT), complete blood count, hematocrit, ethyl glucuronide in urine), and standardized questionnaire forms (circumstances of poisoning, medical history, comorbidities, etc.).

The patients were retrospectively divided into three groups according to the outcome: group I, the patients who survived without sequelae; group II, the patients who survived with sequelae; group III, the patients who died. Patients were considered to have visual impairment if the symptoms of toxic neuropathy of the optic nerve were documented on admission/during hospitalization, with pathologic findings on visual acuity, visual fields, color vision, and contrast sensitivity, and persisting lesions on fundoscopy, OCT, VEP with other symptoms of visual damage were found on discharge from hospital and/or 3-6 months after discharge. Patients were considered as having impairment of the central nervous system (CNS) with sequelae of poisoning if symmetrical necrosis and hemorrhage of the basal ganglia were present on computed tomogram during the hospital stay and/or magnetic resonance image of the brain 3-6 months after discharge.

\section{Treatment}

All patients were treated in accordance to the American Association of Clinical Toxicology and European Association of Poisons Centres and Clinical Toxicologists (AACT/EAPCCT) practice guidelines on the treatment of methanol poisoning ${ }^{26}$. Bicarbonate $8.4 \%$ or $4.2 \%$ solution was administered intravenously as a buffer to patients with metabolic acidosis. Fomepizole (Fomepizole EUSA, EUSA Pharma, France) was administered as a bolus dose of $15 \mathrm{mg} / \mathrm{kg}$ i.v. diluted in isotonic saline, and then $10 \mathrm{mg} / \mathrm{kg}$ every $12 \mathrm{~h}$ in patients without hemodialysis, and every $4 \mathrm{~h}$ during hemodialysis. From the fifth dose and on, $15 \mathrm{mg} / \mathrm{kg}$ was administered in order to compensate for increased metabolism ${ }^{27}$.

Because there was limited availability of fomepizole, the following antidote-saving approach was used: a) if fomepizole was not available immediately, the standard scheme of ethanol administration to rapidly achieve the protective serum concentration 100-150 mg/dL (21.7-32.6 $\mathrm{mmol} / \mathrm{L}$ ) was initiated as soon as possible; b) fomepizole treatment was prioritized in patients with serum methanol higher than $50 \mathrm{mg} / \mathrm{dL}$ ( $15.6 \mathrm{mmol} / \mathrm{L}$ ) (or formate higher than $40 \mathrm{mg} / \mathrm{dL}(8.9 \mathrm{mmol} / \mathrm{L}))$ and $\mathrm{pH} \leq 7.0$, or methanol higher than $30 \mathrm{mg} / \mathrm{dL}(9.4 \mathrm{mmol} / \mathrm{L})$ and $\mathrm{pH} \leq 7.0 \mathrm{in}$ patients unable to hyperventilate $\left(\mathrm{pCO}_{2}>3.07 \mathrm{kPa}\right.$ or $23.0 \mathrm{mmHg}$ ); c) treatment with fomepizole was stopped and followed by ethanol administration when methanol concentration decreased below $30 \mathrm{mg} / \mathrm{dL}$ (9.4 mmol/L) given a normal $\mathrm{pH}$, or $20 \mathrm{mg} / \mathrm{dL}(6.2 \mathrm{mmol} / \mathrm{L})$ if metabolic acidosis was not yet corrected. The rationale for this approach was to decrease the risk of incomplete ADH blocking by possible fluctuations of ethanol levels in the 
most severely poisoned patients, especially during hemodialysis, and to avoid respiratory depression caused by ethanol in patients hyperventilating to compensate for the acidosis.

Hemodialysis was performed if the patients filled any of the following criteria: serum methanol higher than $50 \mathrm{mg} / \mathrm{dL}(15.6 \mathrm{mmol} / \mathrm{L})$, metabolic acidosis with a $\mathrm{pH}<7.30$, or had visual toxicity ${ }^{21}$. The mode of dialysis, intermittent hemodialysis (IHD), extended daily dialysis (EDD), or continuous veno-venous hemofiltration/hemodialysis/hemodiafiltration (CVVH/HD/HDF), was based on several factors, such as the hemodynamic stability of the patient on admission, or the severity of poisoning, but availability also played an important role: some smaller hospitals only had CVVH/HD/HDF available in the anesthesiology departments, whereas larger hospitals usually also had EDD/IHD available.

Folates were administered to substitute the inner pool of tetrahydrofolate: folinic acid (Calcium folinate Hospira, amp. $20 \mathrm{~mL}, 10 \mathrm{mg} / \mathrm{mL}$ Hospira UK Limited, Great Britain, and folic acid (Acidum folicum Léčiva, tbl. $10 \mathrm{mg}$, Zentiva, Czech Republic)). The dose of folinic acid was $50 \mathrm{mg}$, administered intravenously, every 4-6 $\mathrm{h}$ until methanol and formate were eliminated. Folinic acid was diluted $5 \%$ glucose in water and administered over 30-60 min. If folinic acid was not available, $50 \mathrm{mg}$ of folic acid in tablets were administered orally every 3-4 h. Corticosteroids were not administered to patients with visual disturbance.

\section{Laboratory investigations}

Methanol was measured using gas chromatography with flame ionization detection and a direct injection with internal standard (Gas Chromatograph Chrom 5, Laboratory Instruments Prague, Czech Republic), limit of detection $1.9 \mathrm{mmol} / \mathrm{L}(60 \mathrm{mg} / \mathrm{L})$ and day-to-day coefficient of variation $2.5-5.4 \%$. Calibrators and controls were made by dilution of methanol p.a. (Penta, Czech Republic).

Formate was measured enzymatically by a Hitachi analyzer (Hitachi 912, Hitachi Science Systems Ltd., Japan) using formate dehydrogenase (Roche, France) and nicotinamide adenine dinucleotide (NAD) (Roche, France), according to a previously published method ${ }^{28-31}$. Pure sodium formate (Sigma-Aldrich, USA) was used to prepare a standard of $1.1 \mathrm{mmol} / \mathrm{L}$ phosphate buffer and two control sera. Day-to-day coefficient of variation was $5.6 \%$, and the upper reference limit was $0.4 \mathrm{mmol} / \mathrm{L} \mathrm{(20}$ $\mathrm{mg} / \mathrm{L})$.

Serum ethanol was analyzed by gas chromatography with flame ionization detection and a direct injection with internal standard (Gas Chromatograph Chrom 5, Laboratory Instruments Prague, Czech Republic). Limit of detection is $0.9 \mathrm{mmol} / \mathrm{L}(40 \mathrm{mg} / \mathrm{L})$ and day-to-day coefficient of variation $3.8-7.1 \%$. Ethanol standards were purchased from Erba Lachema, Czech Republic. Osmolality was measured by freezing point depression method on a Fiske one-ten osmometer. Reference range for the osmolal gap was -9 - $19 \mathrm{mOsm} /\left(\mathrm{kg} \mathrm{H}_{2} \mathrm{O}\right)$. The osmolal contribu- tion from ethanol was subtracted from the measured osmolality.

\section{Calculations and data analysis}

The admission laboratory data, clinical symptoms, treatment measures and outcomes in the separate groups were compared group by group using Two-Sample Assuming Unequal Variances (Equal Means), Twosample F-Test for Variances, Bias test, and two-sample Kolmogorov-Smirnov test. The normality of data distribution was characterized using skewness and kurtosis tests. Data are expressed as medians and arithmetic means with either range or standard deviation or confidence interval ( significance level $\alpha=0.05$ ), as appropriate. For comparison of results, common statistical tests were (t-Test: TwoSample Assuming Equal Variances, t-Test: Two-Sample Assuming Unequal Variances (Equal Means), Two-sample F-Test for Variances, Bias test, ANOVA test, etc.). All statistical calculations were carried out at a level of significance $\alpha=0.05$ (ref. $^{32,33}$ ). The calculations were performed using Excel 2003 (Microsoft, USA) and QC Expert software 3.1 (Trilobyte, Pardubice, Czech Republic).

\section{RESULTS}

The discharge reports and standardized admission protocols of all 25 patients, median age 50 (16-73 years), 18 males and 7 females, treated with fomepizole were analyzed in Czech TIC. Six patients (24\%) died in hospital and $19(76 \%)$ survived. Eleven of these signed the informed consent and were subjected to clinical examinations three to six months after discharge. The ingested toxic spirits contained approximately 50\% methanol and $50 \%$ ethanol in different kinds of strong alcoholic beverages (Slivovitz, Rum Tuzemak, Vodka Drak and others) with total alcohol content around 40\% ABV (alcohol by volume, v/v). Four subjects consumed other alcoholic beverages (wine, beer, whisky, and home-made spirits) concomitantly. Eight patients had detectable ethanol before hospital treatment with antidote. Only $24 \%$ of the patients were admitted within $24 \mathrm{~h}$ after the methanol ingestion, $40 \%$ within $48 \mathrm{~h}$, and 24\% later than $48 \mathrm{~h}$. In $12 \%$ of cases, it was impossible to identify reliably the time to diagnosis. A medical history of chronic alcohol abuse was present in 6 cases.

\section{Admission data}

The laboratory data in the patients treated with fomepizole on admission are shown in Table 1.

\section{Clinical symptoms}

The clinical features on admission are shown in Table 2.

Among the patients treated with fomepizole 3/25, (12\%) were asymptomatic on admission; two of them appeared inebriated with measurable ethanol in the blood. Among the symptomatic patients, the most frequent signs 


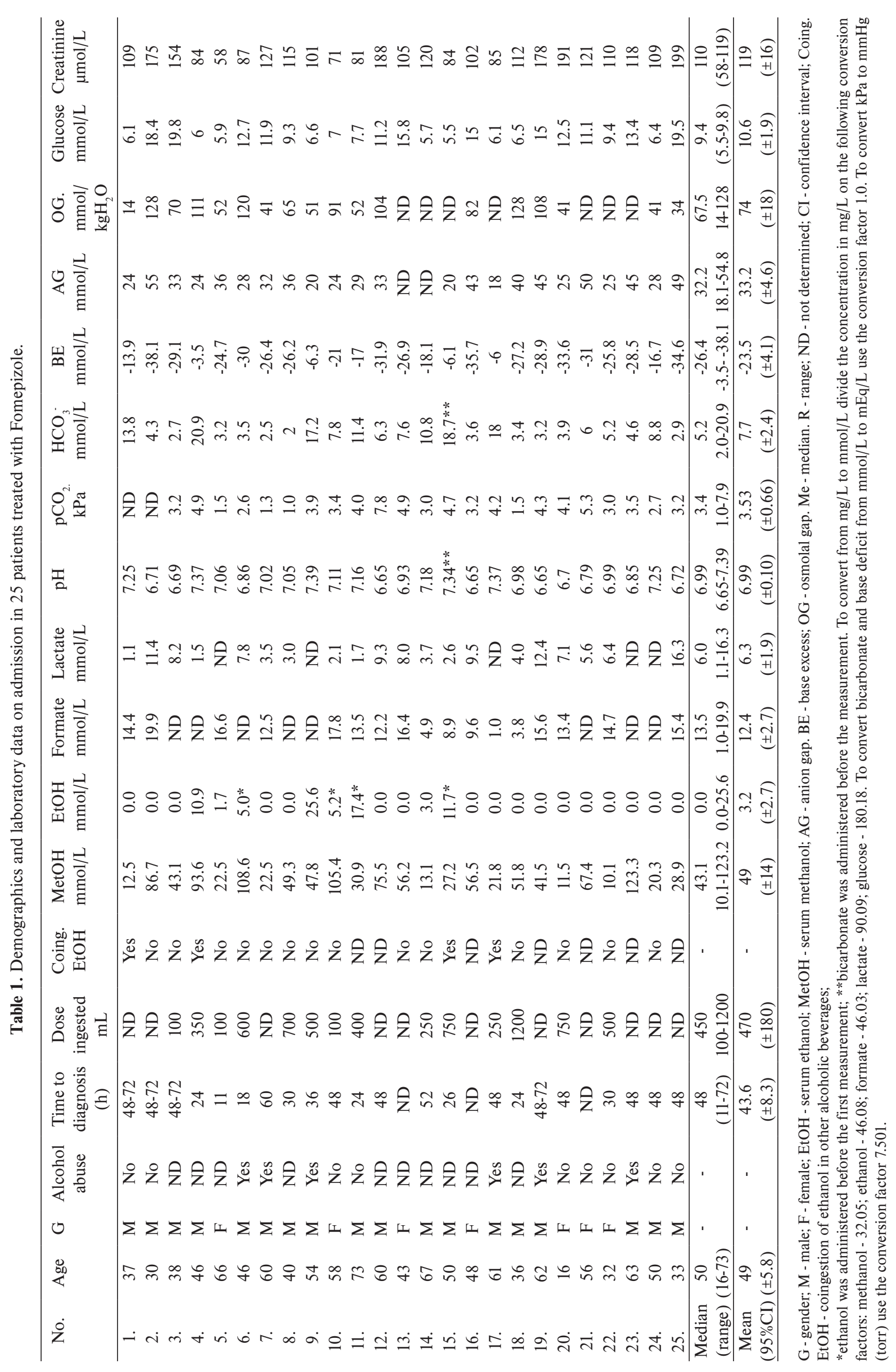




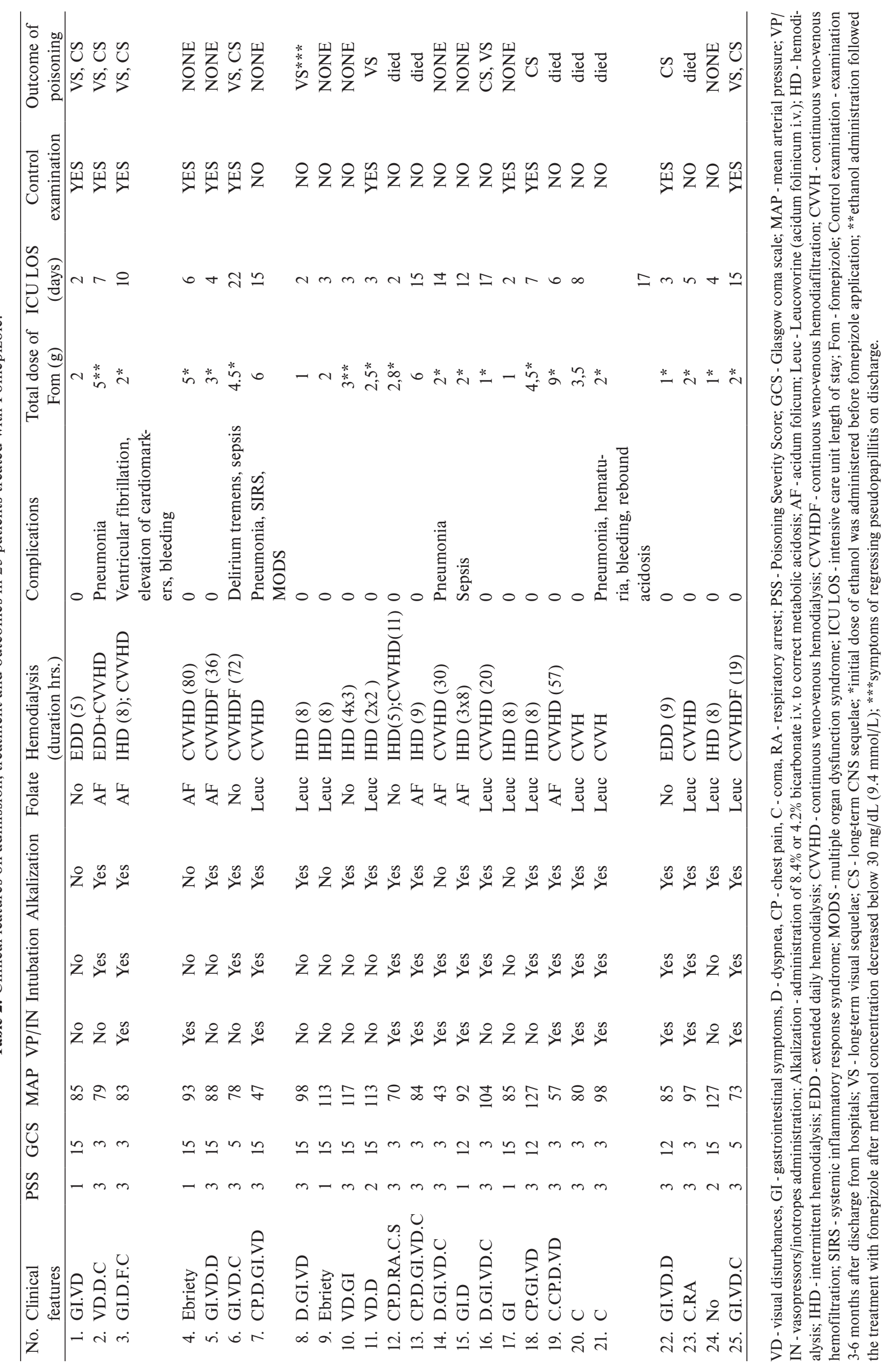


were visual (blurry or cloudy vision, central visual field defects, and alterations in light, color and depth perception) and gastrointestinal disturbance (nausea, upset stomach, vomiting) - both were present in $15 / 25$ patients $(68 \%)$; dyspnea was found in $13 / 25$ (52\%), coma in $12 / 25(48 \%)$, and chest pain in $5 / 25(20 \%)$ patients. Altogether $16 / 25$ (64\%) of the patients were intubated, 13/25 (52\%) were administered vasopressors/inotropes to support hemodynamics, and 18/25 (72\%) of the patients had severe grade of Poisoning severity score (PSS 3 ) on admission.

\section{Treatment}

The treatment of patients with fomepizole included alkalization in $80 \%$; initial bolus of ethanol was administered before fomepizole application in 16/25 (64\%) of cases, and administration of ethanol followed the treatment with fomepizole after the decrease in methanol concentration under $20 \mathrm{mg} / \mathrm{dL}(6.2 \mathrm{mmol} / \mathrm{L})$ and correction of metabolic acidosis in $2 / 25(8 \%)$ cases. The median total dose of fomepizole administered during the course of treatment was $2 \mathrm{~g}$ ( $1-9 \mathrm{~g})$ that is 20 ampules containing $100 \mathrm{mg}$ of the substance. Folates were administered in $20 / 25(80 \%)$ subjects (folinic acid in 12 patients, folic acid in 8 patients). Continuous modes of hemodialysis were applied in $56 \%$ of patients; intermittent hemodialysis was performed in $44 \%$, and extended daily hemodialysis in $12 \%$.

Complications during treatment were found in $7 / 25$ (28\%) of cases: the most frequent being aspiration pneumonia ( 4 cases), followed by sepsis ( 2 cases), and bleeding due to heparinization during the hemodialysis (2 cases). No cases of seizures, hypoglycemia, or pancreatitis, renal or hepatic failure due to fomepizole administration occurred. One case of malignant arrhythmia (ventricular tachycardia with ventricular fibrillation requiring two series of defibrillation) followed by acute coronary syndrome with significant elevation of cardio markers was observed during the IHD. One case of delirium tremens occurred in a patient with a history of chronic alcohol abuse. Finally, one case of rebound of metabolic acidosis after the discontinuation of CVVH due to bleeding episodes occurred.

\section{Outcomes}

The outcomes in the patients treated with fomepizole were the following: $24 \%$ fatalities, $36 \%$ survivors without health impairment, and 40\% survivors with sequelae, of whom solely visual impairment was diagnosed in $2 / 25$ (8\%), solely CNS impairment in $2 / 25$ (8\%), and both visual and CNS sequelae in 6/25 (24\%).

All the patients who died were comatose on admission. Mortality was $50 \%$ among patients in a coma on admission. The patients who survived with health impairment were comatose on admission in $50 \%$ of cases and had signs of visual toxicity before admission in $90 \%$ cases.

Median intensive care unit length of stay (ICU LOS) was six days (range 2-22 days). The median ICU LOS in the patients who survived without impairment was 4 (2-15 days), in those who survived with sequelae 7 (2-22) days, and finally in those who died 7 (2-17) days. One patient with complications during therapy died, three survived with both visual and CNS injury, and other three subjects survived without impairment.

The outcomes of the treatment with fomepizole in three groups of patients are shown in Table 3. Patients who survived without health impairment differed significantly from the other 2 groups in arterial blood $\mathrm{pH}$, bicarbonates, base deficit, anion gap, serum lactate and glucose. The patients who survived with visual/CNS impairment differed from those who died, in serum ethanol on admission and $\mathrm{pCO}_{2}$, but not in severity of metabolic acidosis. There were no significant differences in serum methanol or formate between the three groups.

\section{DISCUSSION}

Methanol poisonings have a high mortality and incidence of long-term health impairment in spite of a complex and resource-consuming treatment ${ }^{34}$. Fomepizole administration as a first-choice antidote is a well-established treatment in patients with acute methanol poisoning $^{14,34}$. However, the relative efficacy of fomepizole is still under discussion ${ }^{35,36}$.

In our study, fomepizole was administered to patients hospitalized during the outbreak of mass methanol poisonings in the Czech Republic. However, due to the limited availability of this antidote, ethanol was used more frequently but the severely poisoned patients were mainly treated with fomepizole. More than $70 \%$ of these patients had a PSS 3 grade and half of them were comatose on admission. Nevertheless, our data demonstrated that the hospital mortality of the patients treated with fomepizole did not differ significantly from the total hospital mortality of all the patients treated in hospitals with acute methanol poisonings in the Czech Republic $(24 \%(n=6 / 25)$ vs. $21 \%(\mathrm{n}=21 / 101), P>0.05))\left(\right.$ ref. $\left.^{24}\right)$. This fact can indirectly suggest that giving fomepizole to the most severely poisoned patients in the resource-limited situation of mass outbreak was justified; the rate of mortality in the most severely poisoned patients treated with fomepizole was no higher than the total hospital mortality rate during the mass methanol outbreak.

The hospital mortality rate in the first mass methanol outbreak in Norway, where fomepizole was used predominantly, did not differ from the hospital mortality rate in our study $(7 / 36,19 \%$ vs. $6 / 25,21 \% ; P>0.05)\left(\right.$ ref. $\left.^{2}\right)$. No other data from mass methanol outbreaks, where fomepizole was used, were found in the literature till now. In Estonia, where solely ethanol was used as an antidote during the outbreak in 2001, reported hospital mortality rate did not differ from the results of our study (23\% vs. $21 \% ; P>0.05)$. Therefore, no direct proof of the greater efficacy of fomepizole with regards to the hospital mortality was found.

Nevertheless, we did not design this study as a randomized control trial, as the groups were likely too small, and fomepizole was often given after the initiation of treat- 


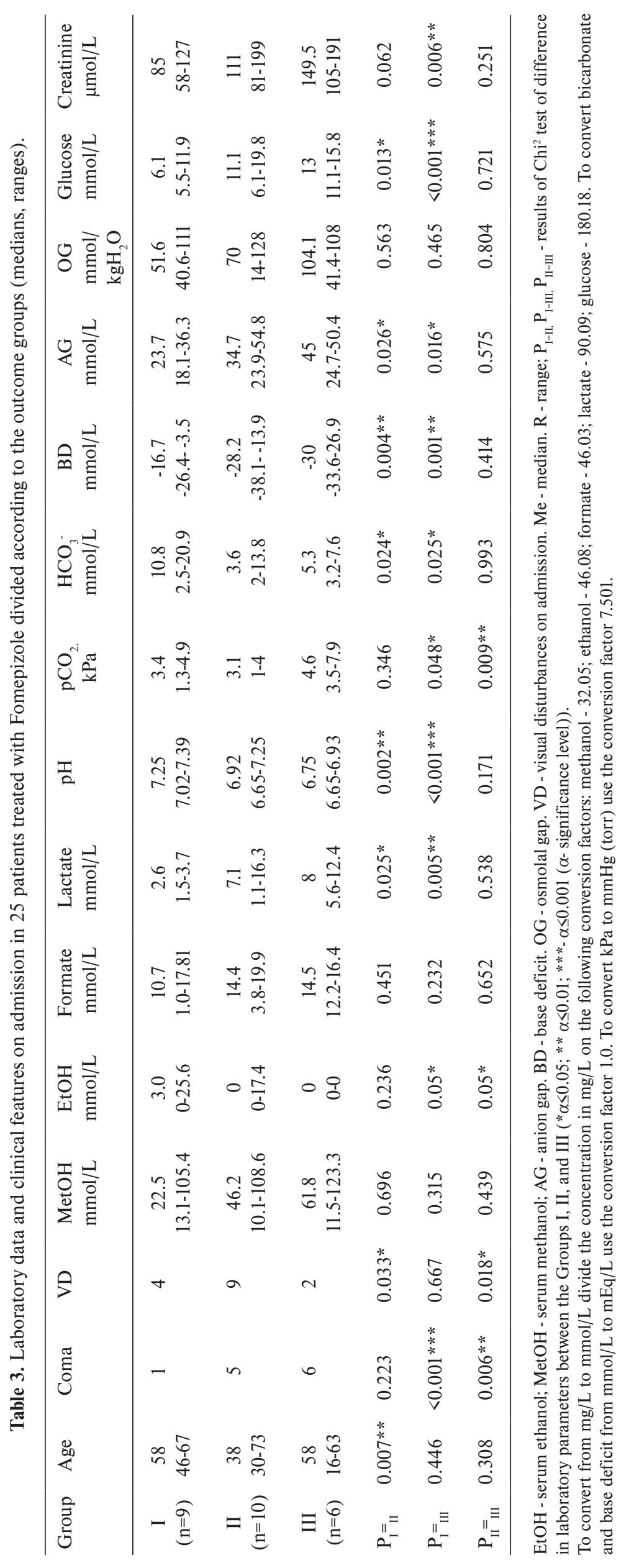


ment with bolus of ethanol or followed by ethanol infusions after the critical phase of poisoning. The benefits of fomepizole observed in the study were indirect ones: no need to monitor serum ethanol level (each 1-2 hours) during the hemodialysis in severely poisoned patients and less work overload on ICU doctors treating several poisoned patients simultaneously. This allowed for management of the most severely poisoned patients through the most critical phases, including the dialysis sessions where ethanol dosing is especially difficult ${ }^{37}$.

The relatively higher prevalence of visual and CNS sequelae in our study compared to the literature, where $18-30 \%$ have been reported ${ }^{26,36}$, can be explained as an underestimation of the long-term impairment due to the absence of follow-up prospective studies in patients who survived poisonings with complete ocular and neurological examinations, and CT/MRI of brain to detect the subclinical damage to the basal ganglia.

In our study we found no adverse effect directly related to the administration of fomepizole. Most complications during treatment were caused by infectious agents and heparinization during the continuous enhanced elimination. No case of hypoglycemia, seizures, pancreatitis, renal or hepatic damage due to administration of fomepizole was reported. The single case of rebound of metabolic acidosis was related to the early discontinuation of CVVH due to bleeding complications and further inhibition of mitochondrial respiration by the remaining formic acid resulting in further accumulation of lactic acid.

One case of delirium tremens was caused by the abrupt discontinuation of ethanol intake in a patient with a history of chronic ethanol abuse of approximately $1 \mathrm{~L}$ of strong alcoholic distillate daily. This case may represent the single condition where fomepizole treatment instead of ethanol has to be considered carefully. As a result of the complication during therapy, this patient was in the ICU for longer than all the other patients.

Finally, the episodes of malignant arrhythmia during the IHD requiring repeated defibrillation in one subject were most probably related to the development of acute coronary syndrome with significant elevation of cardio markers in the severely poisoned patient.

Both the patients who died and the patients who survived with health impairment were significantly more acidotic on admission than those who survived without sequelae; but the difference in severity of metabolic acidosis was not significant between those who died and those who survived with sequelae. In an earlier study of Paasma et al., there was a trend to a "positive" shift in morbidity and mortality (i.e., a better outcome) in the fomepizole group relative to the ethanol group regarding the $\mathrm{pH}$, but this difference was not significant ${ }^{38}$. In our study, the patients treated with fomepizole who survived with visual/ CNS injury had a median $\mathrm{pH} 6.92(6.65-7.25, \mathrm{n}=10)$, that was lower than the median pH $7.02(6.65-7.39, n=20)$ of all the patients surviving methanol poisoning with visual/ CNS impairment during the Czech mass methanol outbreak 2012 (ref. ${ }^{24}$ ), but the difference was not significant $(P=0.218)$. Hence the observation of a "positive" shift in the patients treated with fomepizole from lethal outcome toward survival with sequelae remains unproven.

In conclusion, we have to agree with the statement of Beatty L. et al. based on the results of meta-analysis of all the data available to 2012 on the treatment of toxic alcohols poisoning with fomepizole and ethanol, that "until further evidence on the use of ethanol and fomepizole is available, either antidote may be considered for $\mathrm{ADH}$ blockade. Clinicians should consider other factors, including cost, efficiency, resource utilization, patient demographic, availability of antidote, and familiarity with the drug when making decisions on the management of toxic alcohol ingestion ${ }^{36 " .}$.

\section{STRENGTH AND LIMITATIONS}

The study has limitations. It is neither a randomized controlled trial nor a cohort trial precluding any direct comparisons. It is a case series, with heterogeneous patients, the reporting of ingestion doses might not be accurate, and timing and quality of interventions were not documented in real time. Other factors may confound the results such as the co-ingestion of different types and quantities of concomitant alcoholic beverages, different temporal patterns of toxic alcohol ingestion, co-morbidities, and so on. Many of these factors could be established with some degree of approximation only.

Despite these limitations, this is to date the most comprehensive data ever presented on the use of fomepizole during a mass methanol outbreak. Most of the essential clinical and laboratory data on admission were collected during the hospitalizations using standardized forms distributed to the hospitals by the TIC during the first weeks of the outbreak. Further, this is the first-ever study to include follow-up examination in almost $60 \%$ of the patients who survived the poisoning, 3-6 months after the discharge using advanced technologies to identify and characterize the long-term visual and CNS sequelae of methanol poisonings. All patients were seen within the follow-up examination at the same medical facility, according to a standardized protocol including complete ophthalmologic and neurological examinations, as well as biochemical and toxicological tests to limit the influence of other confounders.

\section{ACKNOWLEDGEMENTS}

Supported with the Projects of Charles University in Prague P25/1LF/2 and P28/1LF/6, the Project of the Ministry of Health of the Czech Republic 12/14/NAP, and EU Project "Material- technical Research Base for the Diagnostics and Treatment of Environmentallycaused and Oncological Disorders and their Risks, in the General University Hospital in Prague" (reg. No. CZ.2.16/3.1.00/24.12).

The study was approved by the General University Hospital Ethics Committee in Prague, Czech Republic. 
Authorship contributions: SZ: data collection, data analysis, data interpretation, manuscript writing; TN: figures, statistical analysis; DP: literature search, study design, critical review of the manuscript.

Conflict of interest statement: The authors state that there are no conflicts of interest regarding the publication of this article.

\section{REFERENCES}

1. Paasma R, Hovda KE, Tikkerberi A, Jacobsen D. Methanol mass poisoning in Estonia: outbreak in 154 patients. Clin Toxicol 2007;45:1527.

2. Hovda KE, Hunderi OH, Tafjord AB, Dunlop O, Rudberg N, Jacobsen D. Methanol outbreak in Norway 2002-2004: epidemiology, clinical features and prognostic signs. J Intern Med 2005;258:181-90.

3. Zakharov S, Navratil T, Pelclova D. Non-fatal suicidal self-poisonings in children and adolescents over a 5-year period (2007-2011). Basic Clin Pharmacol Toxicol 2013;112:425-30. doi: 10.1111/bcpt.12047

4. Megarbane B, Borron SW, Baud FJ. Current recommendations for treatment of severe toxic alcohol poisonings. Intensive Care Med 2005;31:189-95.

5. Zakharov S, Nurieva O, Navratil T, Diblik P, Kuthan P, Pelclova D. Acute methanol poisonings: Folates administration and visual sequelae. J Appl Biomed 2014;12(4):309-16. doi: dx.doi.org/10.1016/ j.jab.2014.04.001

6. Liesivuori J, Savolainen H. Methanol and Formic-Acid Toxicity Biochemical-Mechanisms. Pharmacol Toxicol 1991;69:157-63.

7. Hovda KE, Hunderi OH, Rudberg N, Froyshov S, Jacobsen D. Anion and osmolal gaps in the diagnosis of methanol poisoning: clinical study in 28 patients. Intensive Care Med 2004;30:1842-6.

8. Sejersted OM, Jacobsen D, Ovrebo S, Jansen H. Formate Concentrations in Plasma from Patients Poisoned with Methanol. Acta Med Scand 1983;213:105-10.

9. Vaneckova M, Zakharov S, Klempir J, Ruzicka E, Bezdicek O, Liskova I, Diblik P, Miovsky M, Hubacek JA, Urban P, Ridzon P, Pelclova D, Burgetova A, Masek M, Seidl Z. Methanol Intoxication on Magnetic Resonance Imaging. Cesk Slov Neurol N 2014;77:235-9.

10. McMartin KE, Ambre JJ, Tephly TR. Methanol Poisoning in HumanSubjects - Role for Formic-Acid Accumulation in the MetabolicAcidosis. Am J Med 1980;68:414-8.

11. Zakharov $S$, Pelclova D, Navratil T, Belacek J, Kurcova I, Komzak O, Salek T, Latta J, Turek R, Bocek R, Kucera C, Hubacek JA, Fenclova Z, Petrik V, Cermak M, Hovda KE. Methanol and formate elimination half-life during treatment for methanol poisoning: intermittent haemodialysis vs. continuous haemodialysis/ haemodiafiltration. Kidney Int 2014;86:199-207. doi:10.1038/ki.2014.60

12. Bergeron R, Cardinal J, Geadah D. Prevention of Methanol Toxicity by Ethanol Therapy. N Engl J Med 1982;307:1528.

13. Hantson $P$, Wittebole $X$, Haufroid V. Ethanol therapy for methano poisoning: duration and problems. Eur J Emerg Med 2002;9:278-9.

14. Jacobsen D, Jansen $H$, Wiik-Larsen E, Bredesen JE, Halvorsen S. Studies on methanol poisoning. Acta Med Scand 1982;212:5-10.

15. Baud FJ, Bismuth C, Garnier R, Galliot M, Astier A, Maistre G, Soffer M. 4-Methylpyrazole May Be an Alternative to Ethanol Therapy for Ethylene-Glycol Intoxication in Man. J Toxicol-Clin Toxicol 1986;24:463-83.

16. Burns MJ, Graudins A, Aaron CK, McMartin K, Brent J. Treatment of methanol poisoning with intravenous 4-methylpyrazole. Ann Emerg Med 1997;30:829-32

17. Bekka R, Borron SW, Astier A, Sandouk P, Bismuth C, Baud FJ. Treatment of methanol and isopropanol poisoning with intravenous fomepizole. J Toxicol-Clin Toxicol 2001;39:59-67.

18. Brent J, McMartin K, Phillips S, Aaron C, Kulig K. Fomepizole for the treatment of methanol poisoning. N Engl J Med 2001;344:424-9.
19. Brent J. Fomepizole for ethylene glycol and methanol poisoning. $\mathrm{N}$ Engl J Med 2009;360:2216-23.

20. Brent J. Fomepizole for the treatment of pediatric ethylene and diethylene glycol, butoxyethanol, and methanol poisonings. Clin Toxicol 2010;48:401-6.

21. Hovda KE, Jacobsen D. Expert opinion: fomepizole may ameliorate the need for hemodialysis in methanol poisoning. Hum Exp Toxicol 2008;27:539-46.

22. Megarbane $B$, Borron SW, Trout $H$, Hantson $P$, Jaeger $A$, Krencker $E$, Bismuth C, Braud FJ. Treatment of acute methanol poisoning with fomepizole. Intensive Care Med 2001;27:1370-8.

23. Borron SW, Megarbane B, Baud FJ. Fomepizole in treatment of uncomplicated ethylene glycol poisoning. Lancet 1999;354:831.

24. Zakharov $S$, Pelclova D, Urban $P$, Navratil T, Ridzon $P$, Fenclova Z, Petrik V, Diblik P, Kuthan P, Hubacek JA, Miovsky M, Klempir J, Vaneckova M, Seidl Z, Pilin A, Kotikova K, Nurieva O, Rulisek J, Komarc M, Hovda KE. Czech Mass Methanol Outbreak 2012: Epidemiology, Challenges and Clinical Features. Clin Toxicol 2014: In Press. doi 10.3109/15563650.2014.974106

25. Zakharov S, Pelclova D, Diblik P, Urban P, Kuthan P, Nurieva O, Kotikova K, Navratil T, Komarc M, Seidl Z, Vaneckova M, Hubacek JA, Klempir J, Yurchenko M, Ruzicka E, Miovsky M, Janikova B, Hovda $\mathrm{KE}$. Long-term visual damage after acute methanol poisonings: Prospective study in 50 patients. Ophthalmology 2014: Submitted.

26. Barceloux DG, Bond GR, Krenzelok EP, Cooper H, Vale JA. American Academy of Clinical Toxicology practice guidelines on the treatment of methanol poisoning. J Toxicol-Clin Toxicol 2002;40:415-46.

27. Jacobsen D, Hovda KE. Methanol. In: Shannon MW, Borron SW Burns MJ, editors. Haddad And Winchester's Clinical Management of Poisoning And Drug Overdose. Philadelphia, USA:WB Saunders; 2007; p. 605-11.

28. Hovda $K E$, Urdal $P$, Jacobsen $D$. Increased serum formate in the diagnosis of methanol poisoning. J Anal Toxicol 2005;29:586-8.

29. Hubacek JA, Pelclova D, Seidl Z, Vaneckova M, Klempir J, Ruzicka E, Ridzon P, Urban P, Fenclova Z, Petrik V, Diblik P, Kuthan P, Miovsky M, Janikova B, Adamkova V, Zakharov S. Rare Alleles within the CYP2E1 (MEOS System) could be associated with better short-term Health Outcome after Acute Methanol Poisoning. Basic Clin Pharmacol Toxicol 2014. In Press. doi: 10.1111/bcpt.12310

30. SalekT, Humpolicek P, Ponizil P. Metabolic disorders due to methanol poisoning. Biomed Pap Med Fac Univ Palacky Olomouc Czech Repub 2013 Sep 27. doi: 10.5507/bp.2013.074. [Epub ahead of print]

31. Zakharov S, Kurcova I, Navratil T, Salek T, Pelclova D. Is the measurement of serum formate concentration useful in the diagnostics of acute methanol poisoning? A prospective study of 38 patients. Basic Clin Pharmacol Toxicol 2014: In Press. doi: 10.1111/bcpt.12338.

32. Bezdicek O, Klempir J, Liskova I, Michalec J, Vaneckova M, Seid Z, Janikova B, Miovsky M, Hubacek JA, Diblik P, Kuthan P, Pilin A, Kurcova I, Fenclova Z, Petrik V, Navratil T, Pelclova D, Ruzicka E, Zakharov S. Sequelae of methanol poisoning for cognition. Cesk Slov Neurol N 2014;77/110:320-5.

33. Cizkova P, Navratil T, Sestakova I, Yosypchuk B. Verification of applicability of mercury meniscus modified silver solid amalgam electrode for determination of heavy metals in plant matrices. Electroanalysis 2007; 19:161-71.

34. Megarbane B, Borron SW, Baud FJ. Current recommendations for treatment of severe toxic alcohol poisonings. Intensive Care Med 2005;31:189-95.

35. Kerns Wn, Tomaszewski C, McMartin K, Ford M, Brent J. Formate kinetics in methanol poisoning. J Toxicol, Clin Toxicol 2002;40:137-43.

36. Beatty L, Green R, Magee K, Zed P. A systematic review of ethanol and fomepizole use in toxic alcohol ingestions. Emergency Medicine International 2013;2013:14. doi:10.1155/2013/638057

37. Hantson $P$, Haufroid V, Wallemacq P. Formate kinetics in methanol poisoning. Hum Exp Toxicol 2005;24:55-9.

38. Paasma R, Hovda KE, Hassanian-Moghaddam H, Brahmi N, Afshari $R$, Sandvik $L$, Jacobsen D. Risk factors related to poor outcome after methanol poisoning and the relation between outcome and antidotes--a multicenter study. Clin Toxicol 2012;50:823-31. 